\title{
A!
}

This is an electronic reprint of the original article.

This reprint may differ from the original in pagination and typographic detail.

Manninen, Matti; Puska, M. J.; Nieminen, R. M.; Jena, P.

\section{Electronically induced trapping of hydrogen by impurities in niobium}

Published in:

Physical Review B

DOI:

10.1103/PhysRevB.30.1065

Published: 15/07/1984

Document Version

Publisher's PDF, also known as Version of record

Please cite the original version:

Manninen, M., Puska, M. J., Nieminen, R. M., \& Jena, P. (1984). Electronically induced trapping of hydrogen by impurities in niobium. Physical Review B, 30(2), 1065-1068. https://doi.org/10.1103/PhysRevB.30.1065

This material is protected by copyright and other intellectual property rights, and duplication or sale of all or part of any of the repository collections is not permitted, except that material may be duplicated by you for your research use or educational purposes in electronic or print form. You must obtain permission for any other use. Electronic or print copies may not be offered, whether for sale or otherwise to anyone who is not an authorised user. 


\title{
Electronically induced trapping of hydrogen by impurities in niobium
}

\author{
M. Manninen \\ Research Institute for Theoretical Physics, University of Helsinki, \\ 00170 Helsinki, Finland \\ M. J. Puska \\ Laboratory of Physics, Helsinki University of Technology, \\ 02150 Espoo, Finland \\ R. M. Nieminen \\ Department of Physics, University of Jyväskylä, \\ 40100 Jyväskylä, Finland \\ P. Jena \\ Physics Department, Virginia Commonwealth University, \\ Richmond, Virginia 23284
}

(Received 16 January 1984; revised manuscript received 9 April 1984)

\begin{abstract}
The binding energies of hydrogen and its isotopes to substitutional impurities $\mathrm{Ti}, \mathrm{Cr}$, and $\mathrm{V}$ in niobium have been calculated. The hydrogen-metal interaction is based on the effective-medium theory. The wave mechanics of the hydrogenic interstitials are explicitly dealt with, and the lattice distortion created by the hydrogen is incorporated through the method of lattice statics. The difference in the electronic structure between impurity and host atoms is shown to be largely responsible for the binding of hydrogen to the impurities. The results are in agreement with recent inelastic neutron scattering experiments.
\end{abstract}

The properties of dissolved hydrogen in metals ${ }^{1,2}$ are strongly influenced by the presence of lattice defects, such as vacancies, interstitial and substitutional impurities, dislocations, and grain boundaries. While hydrogen atoms in dilute quantities in pure metals remain in solid solution at elevated temperature, they are known to form precipitates of metal hydride at low temperatures. It has recently been found $^{3,4}$ that the formation of the hydride phase in niobium is prevented or hindered by the presence of impurities in the host material. This phenomenon is commonly understood as arising from the trapping of hydrogen by randomly distributed impurities in the host metal.

The high hydrogen mobility especially in bcc metals, even at low temperatures, enables hydrogen to locate the trap sites rather easily. The origin of this trapping is not well understood in detail. The prevailing hypothesis is that the strain fields arising from the lattice distortion due to the impurity is responsible for hydrogen trapping. Recent experiments, ${ }^{3,4}$ however, cast doubt on the universality of this explanation. In $\mathrm{Nb}$ substitutional $\mathrm{Cr}$ impurities cause a large change in the average lattice constant ${ }^{5}(\Delta a / \Delta c=-5.69$ $\times 10^{-3} \AA /$ at. \%) whereas the elastic deformation due to $\mathrm{Ti}$ is very $\operatorname{small}^{5}\left(\Delta a / \Delta c=-0.32 \times 10^{-3} \AA /\right.$ at. \% $)$. Experimentally, however, the binding energy of hydrogen to $\mathrm{Ti}$ has been found to be larger than the binding energy to $\mathrm{Cr}$. Clearly this finding is inconsistent with the hypothesis that elastic distortions are major factors in hydrogen trapping. Also the systematic diffusion and trapping studies $^{5}$ of $\mathrm{Al}$ doped with various substitutional impurities have revealed no apparent relation between the trapping properties and the long-range deformation. Another origin of the trapping arises from the changes in the electronic structure and interactions near the substitutional impurity.

In this Rapid Communication we report the first $a b$ initio calculation of the role of electronic structure in hydrogen trapping. Our method is based on a comprehensive theory ${ }^{7}$ where we (i) construct the hydrogen potential energy in terms of the host electron density map using the effective medium theory, (ii) include the hydrogen zero-point motion by solving the appropriate three-dimensional Schrödinger equation, and (iii) calculate the hydrogen-induced lattice distortions by using Green's-function techniques within the framework of lattice statics. The whole procedure is then iterated to self-consistency in the potential-energy surface, hydrogen distribution, and lattice distortions. The procedure is suited for investigations of both extended and self-trapped quantum states of hydrogen, and can also deal with isotope effects from the outset. Here, the method is applied to self-trapped states in $N b \mathrm{Ti}, N b \mathrm{~V}$, and $N b \mathrm{Cr}$ systems. Good agreement is achieved between theory and experiment. Similar calculations are carried out for deuterium $\mathrm{D}$ and the positive muon $\mu^{+}$. The isotopic dependence of the impurity trapping energies is weak, the trapping energies for $\mathrm{H}$ and $\mathrm{D}$ being slightly smaller than for $\mu^{+}$.

It is usually energetically favorable for hydrogen in its ground state to be localized at an interstitial site by displacing the host atoms in its immediate vicinity. Such a polaronlike state is commonly referred to as being self-trapped. In systems containing defects such as impurities and lattice vacancies, hydrogen may be trapped even without inducing local deformations because of the change in the electronic structure around the defect. Trapping usually has a significant effect on hydrogen diffusion. The study of the trapping energy is most useful in understanding properties such as the formation of hydride phases and hydrogen-induced embrittlement.

Sugimoto and $\mathrm{Fukai}^{8}$ were the first to treat interstitial hydrogen as a quantum mechanical particle. Our method is 
different from theirs basically only in the construction of the potential-energy field $V(\vec{r})$ felt by the hydrogen. We use the effective-medium theory ${ }^{9}$ which assumes that $V(\vec{r})$ can be constructed as a functional of the electron density $n(\vec{r})$ of the system without the impurity. This functional is nonlocal and contains volume- or density-dependent terms, i.e., does not reduce to a summation over pairwise interactions between hydrogen and the metal atoms. On the other hand, the functional is not known accurately enough to predict quantities involving energetics with a high precision in, e.g., transition-metal based systems. Nevertheless, the method can seemingly well reproduce ${ }^{10}$ qualitative features and trends across the periodic table.

We construct the electron density $n(\vec{r})$ of the host in terms of a superposition of atomic densities $n_{\text {at }}(\vec{r})$, i.e.,

$$
n(\overrightarrow{\mathrm{r}})=\sum_{\overrightarrow{\mathrm{R}}_{i}} n_{\mathrm{at}}\left(\overrightarrow{\mathrm{r}}-\overrightarrow{\mathrm{R}}_{i}\right) \text {, }
$$

where the sum runs over the atomic positions $\vec{R}_{i}$ in the vicinity of the hydrogen. The impurity enters expression (1) by replacing one of the host atoms in the summation. As discussed below, we believe that this approach, which neglects charge transfer in the defect region, is accurate enough for qualitative purposes. Once $V(\vec{r})$ is constructed from the given atomic configurations and positions $\overrightarrow{\mathrm{R}}_{i}$, the localized hydrogen wave function is solved by using threedimensional numerical relaxation techniques. The forces exerted on the neighboring atoms are then calculated from the derivatives of $V(\vec{r})$ and the square of the hydrogen wave function. The ensuing relaxations are then obtained by using the lattice Green's function given for $\mathrm{Nb}$ by MacGillivray and Sholl. ${ }^{11}$ These give a new set of $\vec{R}_{i}$ and, via Eq. (1) and the effective-medium functional, a new density map and a new potential field. The procedure is then repeated until self-consistency is obtained. For more details of the numerical techniques, the reader is referred to the paper by Puska and Nieminen.

The energy of the self-trapped hydrogen coupled to the lattice is given by

$$
E_{s}=E_{\alpha}+E_{L} \text {, }
$$

where $E_{\alpha}$ is the eigenvalue of the hydrogen Schrödinger equation and $E_{L}$ the energy stored in the lattice deformation. The self-trapping energy gain is defined as

$$
E_{\mathrm{ST}}=E_{\alpha}^{\infty}-E_{s},
$$

where $E_{\alpha}^{\infty}$ is the hydrogen energy in an extended (delocalized) state with periodic boundary conditions and no lattice distortions. Finally, the binding energy of hydrogen to an impurity is

$$
\Delta E=E_{s}^{h}-E_{s}^{i},
$$

where the superscripts $h$ and $i$ denote the clean host and the impurity host, respectively. Impurity trapping of hydrogen occurs if $\Delta E>0$.

We have applied the procedure outlined above to $\mathrm{Nb}$ containing dilute amounts of $\mathrm{V}, \mathrm{Cr}$, and $\mathrm{Ti}$ impurities. These systems were chosen since recent inelastic neutron scattering measurements of hydrogen vibration spectra, performed at different temperatures, reveal the trapping energy of hydrogen to a $\mathrm{Cr}$ impurity to be $105 \mathrm{meV}$. In the case of $\mathrm{Ti}$, the trapping of hydrogen was considerably stronger than in $\mathrm{NbCr}$ since no metal hydride phase separation was ob-

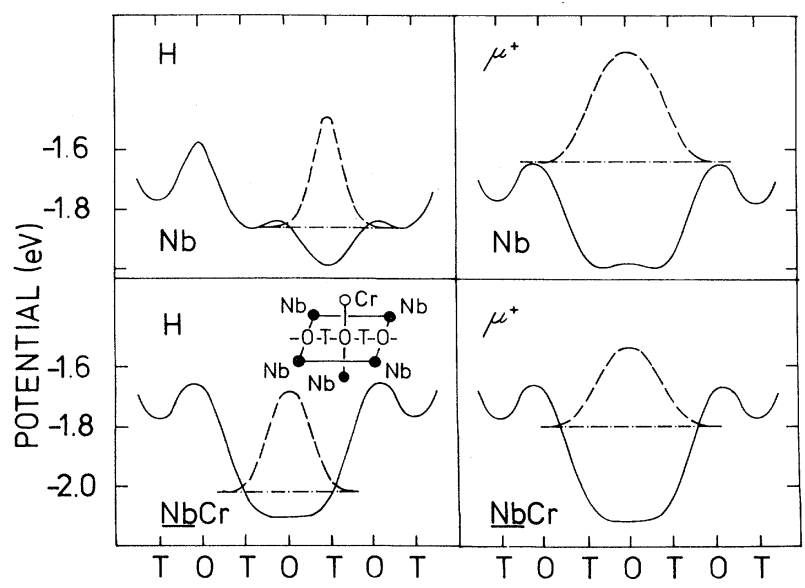

FIG. 1. Stable self-trapped state potential and wave functions for $\mathrm{H}$ (left panel) and $\mu^{+}$(right panel) in pure $\mathrm{Nb}$ (upper row) and in $\mathrm{Nb}$ with a substitutional $\mathrm{Cr}$ impurity (bottom row). The potentials and wave functions are drawn along a straight line passing through the adjacent octahedral and tetrahedral sites as shown in the schematic inset. The potentials are denoted by solid and the wave functions by dashed lines, respectively. The dash-dotted lines give the energy eigenvalues $E$ relative to the potential. In the case of one separated $\mathrm{Cr}$ impurity the stable site is the octahedral site adjacent to the impurity. The geometry is shown in the inset.

served. It was thus concluded that the binding energy of hydrogen to $\mathrm{Ti}$ must be larger than $120 \mathrm{meV}$, which is the enthalpy of formation of the hydride phase. In the case of $N b \mathrm{~V}$ trapping of hydrogen was also observed even though no value for the binding was given.

In Table I we present our results for $E_{\alpha}, E_{L}$, and the self-trapping energy $E_{\mathrm{ST}}$ as well as the lattice displacements of near-neighbor atoms for hydrogenic interstitials in pure $\mathrm{Nb}$. Results are given for both the octahedral $(O)$ and tetrahedral $(T)$ interstitial sites in the bcc structure. For $\mathrm{H}$ and $\mathrm{D}$, the $T$ site has the lowest energy, whereas for $\mu^{+}$the $O$ site is the stable one. For the latter, the wave function extends to the four neighboring $T$ sites and is therefore sometimes given the assignment $4 T$. The situation is further illustrated in Fig. 1(a). The self-trapping is governed by the outward relaxation of the nearest neighbors; the displacement of the second nearest neighbors are already an order of magnitude smaller.

To compute the trapping energy of hydrogen to the impurities we have replaced one of the $\mathrm{Nb}$ atoms closest to the self-trapped hydrogen by the impurity atom. This replacement is done with the displaced positions, i.e., no further lattice relaxation is allowed. In reality, however, the impurity-induced change in the hydrogen wave function and the impurity itself would contribute further to the lattice deformation. These are neglected since at the present stage the effect of the substitutional impurity on the lattice Green's function is not known. Furthermore, we assume that the distortion energy $E_{L}$ is not affected by the substitution. The approximations mean that we focus on the effect of electronic structure alone on hydrogen trapping.

In Table II we present the trapping energies for $\mathrm{H}, \mathrm{D}$, and $\mu^{+}$to $\mathrm{Ti}, \mathrm{V}$, and $\mathrm{Cr}$ impurities in $\mathrm{Nb}$. The binding to the impurity arises mainly from the more compact electron cloud around the impurity as compared to a host atom. 
TABLE I. Results for hydrogenic impurities in pure Nb. $E_{\alpha}$ is the energy eigenvalue of the hydrogenic impurity, $E_{L}$ the lattice strain energy, and $E_{\mathrm{ST}}$ the self-trapping energy gain. All energies are in eV. The relaxations are given for the first two neighboring shells by denoting the position vector of one relaxed atom in units of the lattice constant $a=3.30 \AA$.

\begin{tabular}{|c|c|c|c|c|}
\hline \multirow{2}{*}{\multicolumn{2}{|c|}{$\begin{array}{c}\text { Delocalized state } \\
E_{\alpha}^{\infty}\end{array}$}} & $\mu^{+}$ & $\mathrm{H}$ & $\mathrm{D}$ \\
\hline & & -1.33 & -1.62 & -1.67 \\
\hline \multicolumn{5}{|c|}{ Self-trapped states } \\
\hline \multicolumn{2}{|r|}{$E_{\alpha}$} & -1.62 & -1.87 & -1.90 \\
\hline \multirow[t]{2}{*}{$T$} & $E_{L}$ & 0.14 & 0.10 & 0.10 \\
\hline & $E_{\mathrm{ST}}=E_{\alpha}^{\infty}-E_{\alpha}-E_{L}$ & 0.16 & 0.15 & 0.14 \\
\hline \multirow{2}{*}{\multicolumn{2}{|c|}{ Relaxations I }} & $(0.5243,0,0.2569)$ & $(0.5207,0,0.2574)$ & $(0.5201,0,0.2573)$ \\
\hline & & $(0.4989,0,0.7483)$ & $(0.4988,0,0.7480)$ & $(0.4988,0,0.7481)$ \\
\hline & $E_{\alpha}$ & -1.64 & -1.87 & -1.90 \\
\hline \multirow[t]{2}{*}{$O$} & $E_{L}$ & 0.15 & 0.12 & 0.12 \\
\hline & $E_{\mathrm{ST}}=E_{\alpha}^{\infty}-E_{\alpha}-E_{L}$ & 0.17 & 0.13 & 0.12 \\
\hline \multirow{2}{*}{\multicolumn{2}{|c|}{$\begin{array}{l}\text { Relaxations I } \\
\text { II }\end{array}$}} & $(0.5394,0,0)$ & $(0.5369,0,0)$ & $(0.5364,0,0)$ \\
\hline & & $(0.5025,0.5025,0)$ & $(0.5012,0.5012,0)$ & $(0.5011,0.5011,0)$ \\
\hline
\end{tabular}

This tendency follows from the prediction of the effectivemedium theory that hydrogen will favor interstitial regions of relatively low electron density. Thus, in the present context a quite general result is that impurity atoms smaller than the host atoms have a tendency to trap hydrogen, whereas larger atoms will repel. This observation is valid as regards the electronic contribution to binding: the impurityinduced lattice deformation will generally favor trapping, as is evidenced by, e.g., the observed hydrogen localization at interstitial impurities $(\mathrm{N}, \mathrm{O})$ in $\mathrm{Nb}$.

The trapping of hydrogen to a $\mathrm{Cr}$ impurity is illustrated in Fig. 1(b). One should note that the stable trapping site for $\mathrm{H}$ and $\mathrm{D}$ is the $O$ position next to the impurity, instead of the $T$ site in pure $\mathrm{Nb}$. This is due to the smaller number of nearest neighbors (2) in the $O$ site than in the $T$ site (4): the relative effect of the impurity-induced change in lowering the electron density sampled by hydrogen is thus larger in the $O$ site.

This site assignment disagrees with the experimental inter-

TABLE II. Results for the trapping to substitutional impurities in Nb. $E_{\alpha}$ is the energy eigenvalue of the hydrogenic impurity and $\Delta E$ the binding energy to the impurity. The lattice strain energy is taken from the pure metal calculation (Table I). All energies are in $\mathrm{eV}$.

\begin{tabular}{lcrrrr}
\hline \hline Impurity & Site & & \multicolumn{1}{c}{$\mu^{+}$} & \multicolumn{1}{c}{$\mathrm{H}$} & \multicolumn{1}{c}{$\mathrm{D}$} \\
\hline \multirow{3}{*}{$\mathrm{Ti}$} & $O$ & $E_{\alpha}$ & -1.78 & -2.00 & -2.03 \\
& & $\Delta E$ & 0.13 & 0.11 & 0.11 \\
& $T$ & $E_{\alpha}$ & -1.74 & -1.97 & -2.00 \\
& & $\Delta E$ & 0.11 & 0.10 & 0.10 \\
$\mathrm{~V}$ & $O$ & $E_{\alpha}$ & -1.78 & -2.00 & -2.00 \\
& & $\Delta E$ & 0.13 & 0.11 & 0.11 \\
& $T$ & $E_{\alpha}$ & -1.74 & -1.97 & -2.00 \\
& & $\Delta E$ & 0.11 & 0.10 & 0.10 \\
$\mathrm{Cr}$ & $O$ & $E_{\alpha}$ & -1.80 & -2.02 & -2.05 \\
& & $\Delta E$ & 0.16 & 0.14 & 0.13 \\
& $T$ & $E_{\alpha}$ & -1.77 & -2.00 & -2.02 \\
& & $\Delta E$ & 0.14 & 0.13 & 0.12 \\
\hline \hline
\end{tabular}

pretation $^{3}$ based on the intensity and energy ratios of the neutron energy loss peaks. However, the calculated energy differences in the trapping to $O$ and $T$ sites is so small $(\sim 20 \mathrm{meV})$ that no definite prediction for lowest position can be made before the full lattice relaxation due to the impurity is included. We also note that the binding energy $\Delta E$ to the impurity is practically independent of the isotope mass. The hydrogen trapping energies are also quite similar for the three impurities considered here.

The results in Table II show that the changes in electronic interactions are large enough to explain experimental observation. This clearly demonstrates that the electronic structure of impurities has substantial influence over hydrogen trapping and that theories based on trapping by elastic deformation alone need to be reexamined. Within the effective-medium theory, electronically induced trapping depends on the difference between the electron densities in pure and impurity-containing systems in the region scanned by zero-point amplitude.

It is gratifying that the calculated trapping energies are of similar magnitude as the experimental ones, particularly since our theory contains no adjustable parameters. However, caution is to be exercised in taking the agreement too seriously; for example, our calculation shows a slightly stronger binding to a $\mathrm{Cr}$ impurity than to $\mathrm{Ti}$ - the opposite of the experimental result. We use the superimposed atomic electron densities, which undoubtedly will change when the interactions between the host metal and impurity atoms are switched on. Secondly, the initial lattice distortion created by the impurities will also affect the quantitative nature of the trapping energies in Table II. What is most significant here is that the electronic structure itself plays a much more important role in hydrogen trapping than hitherto believed. The electronic affinity of hydrogen to $\mathrm{Ti}$, for example, is likely to be a factor in the high solubility of hydrogen in $\mathrm{FeTi}$ alloys which are used for hydrogen storage.

No experiments are yet available to test the predictions of the isotopic independence of binding energies to the trap given in Table II. We hope that such experiments would be performed, as they will shed additional light on the interesting problem of hydrogen trapping in metals. 


\section{ACKNOWLEDGMENTS}

This work was supported in part by grants from Thomas F. Jeffress and Kate Miller Jeffress Memorial Trust and National Science Foundation. We are grateful for the hospitality of NORDITA where this work was initiated. Many useful discussions with Jens Nørskov are gratefully acknowledged.

${ }^{1}$ Hydrogen in Metals I-II, Topics in Applied Physics, edited by G. Alefeld and J. Völkl (Springer, Heidelberg, 1978).

${ }^{2}$ Electronic Structure and Properties of Hydrogen in Metals, edited by P. Jena and Satterthwaite (Plenum, New York, 1983).

${ }^{3}$ D. Richter, J. J. Rush, and J. M. Rowe, Phys. Rev. B 27, 6227 (1983).

${ }^{4}$ A. Magerl, J. J. Rush, J. M. Rowe, D. Richter, and H. Wipf, Phys. Rev. B 27, 927 (1983).

${ }^{5}$ W. B. Pearson, Lattice Spacings and Structure of Metals and Alloys (Pergamon, Oxford, 1958).
${ }^{6}$ K. W. Kehr, D. Richter, J.-M. Welter, O. Hartmann, E. Karlsson, L. O. Norlin, T. Niinikoski, and A. Yaouanc, Phys. Rev. B 26, 567 (1982).

${ }^{7}$ M. J. Puska and R. M. Nieminen, Phys. Rev. B 29, 5382 (1984).

${ }^{8}$ H. Sugimoto and Y. Fukai, Phys. Rev. B 22, 670 (1980).

${ }^{9}$ J. K. Nørskov and N. D. Lang, Phys. Rev. B 21, 2316 (1980), M. J. Stott and E. Zaremba, ibid. 22, 1564 (1980)

${ }^{10}$ J. K. Nørskov, Phys. Rev. B 26, 2875 (1982).

${ }^{11}$ I. R. MacGillivray and C. A. Sholl, J. Phys. F 13 , 23 (1983). 